\title{
Piecewise-Quadrilateral Registration by Optical Flow - Applications in Contrast-Enhanced MR Imaging of the Breast
}

\author{
Michael S. Froh ${ }^{1}$, David C. Barber ${ }^{2}$, Kristy K. Brock ${ }^{3}$, Donald B. Plewes ${ }^{1}$, \\ and Anne L. Martel ${ }^{1}$ \\ ${ }^{1}$ Department of Medical Biophysics, University of Toronto, Canada \\ mfroh@swri.ca \\ ${ }^{2}$ Department of Medical Physics, Central Sheffield Teaching Hospitals, Sheffield, UK \\ ${ }^{3}$ Physics Department, Princess Margaret Hospital, Canada
}

\begin{abstract}
In this paper we propose a method for the nonrigid registration of contrast-enhanced dynamic sequences of magnetic resonance(MR) images. The algorithm has been developed with accuracy in mind, but also has a clinically viable execution time (i.e. a few minutes) as a goal. The algorithm is driven by multiresolution optical flow with the brightness consistency assumption relaxed, subject to a regularized best-fit within a family of transforms. The particular family of transforms we have employed uses a grid of control points and trilinear interpolation. We present validation results from a study simulating non-rigid deformation by a biomechanical model of the breast, with simulated uptake of a contrast agent. We further present results from applying the algorithm as part of a routine breast cancer screening protocol.
\end{abstract}

\section{Introduction}

Dynamic contrast-enhanced MR imaging of the breast has been shown to be a highly sensitive screening technique for the early detection of breast carcinoma in patients at risk for hereditary breast cancer [1. In this context, a baseline volumetric image of the patient is acquired, followed by an injection of a gadolinium diethylenetriamine penta-acetic acid (Gd-DTPA) contrast agent. A series of subsequent volumetric images are acquired and compared to the original baseline volume. In a tumour, Gd-DTPA accumulates in the extravascular, extracellular matrix around a lesion as a result of tumour angiogenesis, and increases the MRI signal due to T1 changes. The enhancing tumour is assessed on the dynamics of contrast medium uptake and morphological features of lesions, which aid in differentiating malignant for benign lesions [2,3]. Subtraction of pre-contrast scans from post-contrast scans significantly increases lesion conspicuity; however, interscan motion will create false positive regions of enhancement and suppress true lesions. While every reasonable effort is made to restrict gross patient motion, movement on the order of pixel spacing $(\sim 1 \mathrm{~mm})$ is common. Thus, an extensive field of research has arisen to find methods to compute and compensate for patient motion in post-processing. 
One of the earliest methods proposed for the registration of contrast-enhanced MR images of the breast was that of Zuo et al. 4] in 1996, in which they attempted to minimize the variance ratio between a pair of images to compute a rigid transformation. Breast motion, however, due to the soft tissues involved, is generally considered to be non-rigid. Kumar et al., also in 1996, devised an optical flow-based method to compute an affine transformation, followed by a single iteration of local flow 5. Standard optical flow techniques rely on the assumption that the intensity of an object remains constant over time, while its position changes, though this assumption fails in the context of contrast-enhanced imaging. In 1997, Davis et al. proposed an "elastic body spline" technique 6], which produced a deformation based on matched control points selected a priori. Rueckert et al. developed a non-rigid registration method using a grid of control points, with displacement of non-grid points determined by cubic B-spline interpolation [7]. This algorithm has been validated against a biomechanical model employing finite-element methods [8]. Unfortunately, this method may require several hours to complete. The algorithm of Rueckert et al. was modified by Rohlfing et al. by the addition of an incompressibility constraint [9], to better handle cases in which the enhancing region may shrink (in order to yield further improvements in mutual information). More recently, Crum et al have proposed a multi-resolution fluid registration algorithm applied to breast MR images, with promising results in terms of execution time and accuracy [10.

Herein, we propose a non-rigid registration algorithm which employs a relaxed formulation from [11] of the classical optical flow equation [12. This formulation introduces a linear "brightness shift" term to account for legitimate changes in signal intensity. Furthermore, to reduce the number of degrees of freedom, and to constrain calculated deformations, the optical flow equations guide the displacement of a grid of control points, with non-grid displacements computed by trilinear interpolation.

\section{Derivation}

We first explain the optical flow equations we use, based on the relaxed formulation of Gennert and Negahdaripour [11. Using a relaxed form is necessary, as the brightness constancy assumption of Horn and Schunck 12 will not hold in the context of contrast-enhanced imaging. In Section 2.2. we reduce the number of degrees of freedom by applying a piecewise transformation governed by a grid of control points. While we apply a linear interpolation between control points, the theory is equally applicable to higher-order transforms (e.g. using cubic B-splines, perhaps).

\subsection{Optical Flow with Linear Brightness Shift}

Recall the optical flow equation of Horn and Schunck [12:

$$
f(x)-m(x)=\Delta \mathbf{x}(x)^{T} \nabla m(x),
$$


where $f(x)$ represents the intensity value at voxel $x$ in the fixed image, $m(x)$ represents the intensity value of voxel $x$ in the moved image, and $\Delta \mathbf{x}(x)$ is the displacement of voxel $x$ between the fixed and moved images. This formulation was generalized by Gennert and Negahdaripour [1] to permit a linear transformation between intensity values between the fixed and moved images. We apply a symmetric form of this relaxed equation at each voxel:

$$
f(x)-m(x)=\frac{1}{2}\left[\Delta \mathbf{x}(x)^{T}(\nabla f(x)+\nabla m(x))-\Delta a(x)(f(x)+m(x))\right] .
$$

In this equation, $\Delta a(x)$ represents a linear shift in voxel intensity with respect to the mean intensity at that position in the fixed and moved images.

\subsection{Piecewise Deformation}

If we apply Equation 2 at every voxel, we may encapsulate the resulting equations with the following linear system:

$$
f-m=R\left(\begin{array}{c}
\Delta \mathrm{x} \\
\Delta \boldsymbol{a}
\end{array}\right) .
$$

Given a matrix $T$ which maps a deformation from a set of parameters, $\boldsymbol{p}$, to the complete space of voxels (including intensity shift), we may write this system as $\boldsymbol{f}-\boldsymbol{m}=R T \boldsymbol{p}$.

While the theory allows any transformation to be used (affecting only the choice of $T$ ), we select as our class of deformations the piecewise-quadrilateral transform: a grid of control points is placed over the image, and individual voxel parameters are computed by linear interpolation between adjacent control points (see e.g. [13]). We typically use a grid-spacing of $12 \mathrm{~mm}$, corresponding to approximately 16 pixels in-plane, and 4-6 slices. The resulting system of equations is

$$
\boldsymbol{f}-\boldsymbol{m}=P\left(\begin{array}{c}
\hat{\Delta} \mathbf{x} \\
\hat{\Delta} \boldsymbol{a}
\end{array}\right)
$$

where $P=R T$ and $(\hat{\Delta \mathbf{x}}, \hat{\Delta} \boldsymbol{a})$ is the vector of control point displacements.

\subsection{Regularization, Normalization, and Preconditioning}

We ensure smoothness of solutions by regularizing the normal equation derived from Equation 4. Thus,

$$
P^{T}(\boldsymbol{f}-\boldsymbol{m})=\left(P^{T} P+\lambda^{2} Q^{T} Q\right)\left(\begin{array}{c}
\hat{\Delta} \mathbf{x} \\
\hat{\boldsymbol{\Delta}} \boldsymbol{a}
\end{array}\right),
$$

where $Q$ is defined to ensure some correlation between displacements of adjacent control points. Specifically, our choice of $Q$ applies a discretized Laplacian operator. 
We wish to ensure that the intensity values of the fixed and moved images are within a similar range. Thus, we normalize the intensity values in each image (by scaling) to yield mean intensities of 1 .

We precondition $P^{T} P$ to account for the incomparability of scale between displacement and intensity shift. We compute a scaling factor for the intensity component of $P$ by first generating an estimate for $P^{T} P$ using only intensity values from the fixed image (i.e. setting $m=0$ ). We compute the trace of the subcomponents of $P^{T} P$ corresponding to the spatial displacements $\left(t_{x}, t_{y}, t_{z}\right)$ and intensity shift $\left(t_{a}\right)$, and scale future computed intensity shift components by $\left(t_{x}+t_{y}+t_{z}\right) / 3 t_{a}$. Further, to ensure consistency of the regularization parameter, we scale $\lambda$ by $\sqrt{\operatorname{tr}\left(P^{T} P\right) / \operatorname{tr}\left(Q^{T} Q\right)}$, using the initial estimate for $P^{T} P$ after rescaling the intensity component. Finally, we solve Equation (5) using a preconditioned biconjugate gradient method (using the diagonal part of $P^{T} P+\lambda Q^{T} Q$ as a preconditioner).

\subsection{Update}

We solve equation (5) at each iteration to compute a new transformation relative to the current estimate. Given the current transformation, $T_{n}$, we compute a new displacement $T_{n+1}^{\prime}$ between $T_{n}(m)$ and $f$. The simplest way to update our transformation would be to set $T_{n+1}=T_{n}+T_{n+1}^{\prime}$. However, this would be inaccurate, as $T_{n+1}^{\prime}$ describes a displacement of control points in the space deformed by $T_{n}$. Thus, the correct update formula is $T_{n+1}=T_{n}+T_{n}^{-1}\left(T_{n+1}^{\prime}\right)$.

Note that the regularization term in equation (5) must also be adjusted to account for the existing displacement (as the regularization should guarantee the smoothness of the composed transformation). In the interest of efficient computation, we sacrifice accuracy here and simply regularize the additive composition (e.g. replacing $\hat{\Delta} \mathbf{x}$ with $\hat{\Delta x}+\Delta \hat{\mathbf{x}}_{\text {old }}$ ).

Following the update, we compute the normalized mutual information (NMI) between our current estimate and the target volume. If the mutual information increases, we accept the new transform. Otherwise, we assume that the smoothness constraint is restricting our ability to find a suitable solution, and so we discard the current estimate, reduce $\lambda$, and solve (5) again. If the mean control point displacement in $T_{n+1}^{\prime}$ falls below a defined threshold, we decrease $\lambda$ (though we keep the current estimate), to avoid slow, asymptotic convergence. If $\lambda$ falls below a defined threshold, the algorithm terminates, returning the last acceptable transformation.

\section{Validation}

We wished to verify objectively that our registration algorithm correctly computes non-rigid deformations between images in a contrast-enhanced sequence. We first simulated a contrast-enhanced sequence with no movement between successive images, shown in Figure 1. Next, we generated a known deformation, which was applied to each image in the sequence. Finally, we registered the non-deformed pre-contrast image in each sequence to each deformed image in 


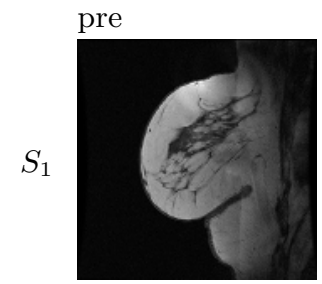

post 1
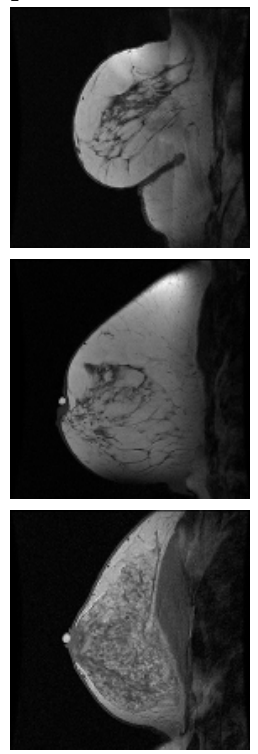

post 2
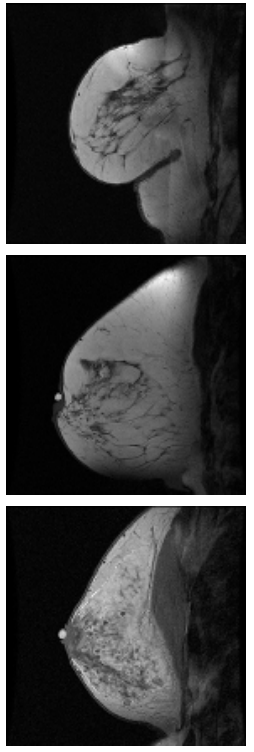

post 2 - pre
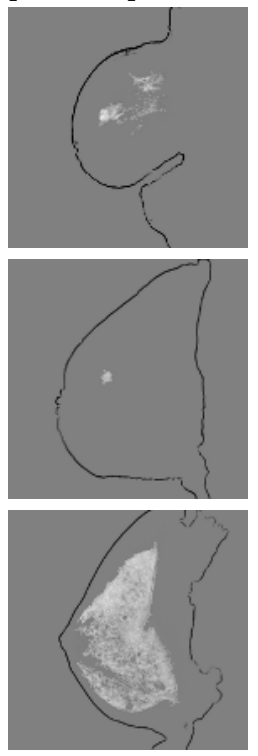

Fig. 1. Simulation studies. For each series, we show a slice from the pre-contrast baseline image, and the two post-contrast images. Contrast change is also shown within an outline of the breast. Volumes of contrast change for sequences $S_{1}, S_{2}$, and $S_{3}$ were approximately $20 \mathrm{~cm}^{3}, 1 \mathrm{~cm}^{3}$, and $280 \mathrm{~cm}^{3}$ respectively.

the sequence, to verify the accuracy of the algorithm in the presence of intensity changes. If the algorithm computes the correct transformation, it should displace each voxel in the same manner as the known deformation.

We simulated physically plausible movement using finite-element methods, employing a biomechanical model of tissue properties. Finite-element methods have been used to produce realistic deformations in breast MR images in [8, for use in the validation of a nonrigid registration technique. Following segmentation of our input images, we labeled the fatty tissue, tumourous carcinoma, fibroglandular tissue, and skin. The Young's moduli for these tissues and the Poisson's ratio were set to the same values as in [8. For each time series, we simulated a point displacement and compression between a pair of mammographic plates.

After registering the data, we were able to compare computed voxel displacements to the true values produced by the biomechanical model. We compared our results to those obtained with a pure affine registration and an existing validated registration algorithm [14]. We used three sets of time series data, each with three time points (one pre-contrast and two post-contrast), and two deformations (point displacement and plate compression), for a total of eighteen volumes to be registered to the pre-contrast, undeformed volumes. Results are summarized in Figure 2 ,

Our algorithm performed well over the eighteen data sets, reducing the mean per-voxel error from approximately $4.2 \mathrm{~mm}$ before registration to approximately 


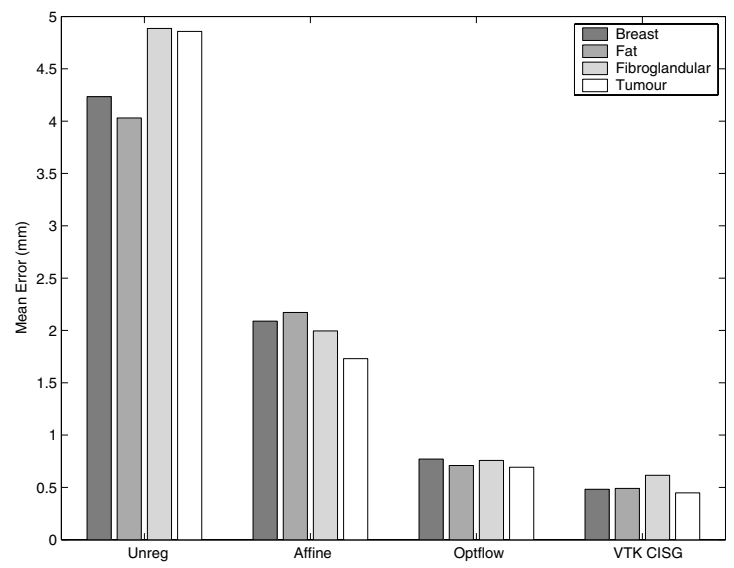

Fig. 2. Mean error per voxel, showing results over the whole breast and for each of the segmented regions (fat, fibroglandular tissue, and tumour tissue). Results shown are average values over the unregistered volumes, and over the volumes following affine registration, registration by our piecewise-quadrilateral optical flow method, and registration by the non-rigid method of Rueckert et al. 7].

$0.77 \mathrm{~mm}$, significantly outperforming the affine registration method (with $2.1 \mathrm{~mm}$ mean error), and obtaining results nearly as accurate at those of 14] (with $0.48 \mathrm{~mm}$ mean error). Furthermore, our algorithm increased the mean correlation coefficient between the source and target volumes 0.8383 to 0.9783 , and reduced the sum of squared differences (SSD) by an average of $63 \%$ (while the method of [14 achieved a mean correlation coefficient of 0.9780 , and an SSD reduction of $68 \%$.) The execution times per volume on a $3 \mathrm{Ghz}$ Intel Pentium 4 workstation were approximately 1 minute, 2 minutes, and 6 hours, for the affine algorithm, the piecewise-quadrilateral algorithm, and [14, respectively.

\section{Clinical Results}

The algorithm presented herein has been applied to over four hundred dynamic contrast-enhanced screening examinations between September 2004 and February 2006. Examining 49 examinations, between December 2005 and February 2006 , with a total of 372 post-contrast volumes, we found that 178 volumes were untouched by the algorithm (as no significant movement was found), while the remainder saw an average $11 \%$ reduction in SSD and an increase in mean correlation coefficient from 0.980 to 0.985 . Furthermore, several cases have been found in which features were undetectable on subtraction images prior to registration, but were clearly visible following registration. One such case is shown in Figure 3. In use, the registration method is straightforward, and may be launched with a single command from the scanner console. Registration is performed on a cluster of eight $1 \mathrm{Ghz}$ Intel Pentium III processors, with eight post-contrast volumes registered in approximately three minutes. 

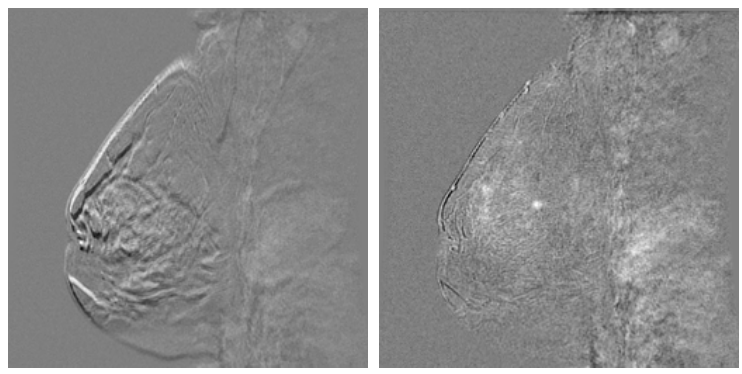

Fig. 3. Subtraction images of a slice acquired from a screening subject before (left) and after (right) registration. Note the small circular region of enhancement near the centre of the breast in the registered image.

\section{Conclusions}

We have presented a fast non-rigid registration algorithm which attempts to overcome some of the traditional limitations of optical flow techniques, while computing accurate transformations in little time. In future, we may experiment with other families of deformations, such as cubic B-splines over a grid of control points. Simulations based on a biomechanical model of breast tissues support our claim that in the presence of contrast enhancement, the algorithm is able to accurately compute a correct deformation to compensate for non-rigid motion of the breast. The algorithm significantly outperformed an affine registration method, yielding accuracy nearly equal to that of an existing validated nonrigid method, in a fraction of the time. The algorithm has been applied to several hundred screening exams, compensating for actual patient motion. Results have been well-received by a collaborating radiologist and our method has successfully exposed areas of enhancement undetectable on unregistered subtraction images. Our experience to date indicates that this is an effective, practical, and timeefficient means to conduct co-registration of dynamic contrast-enhanced MR images for breast screening in the clinic.

\section{Acknowledgments}

We wish to thank Elizabeth Ramsay for assistance in gathering the breast MRI data. We would also like to thank the Canadian Breast Cancer Research Alliance and the Terry Fox Foundation for support in breast MRI research.

\section{References}

1. Warner, E., Plewes, D.B., Hill, K.A., Causer, P.A., Zubovits, J.T., Jong, R.A., Cutrara, M.R., DeBoer, G., Yaffe, M.J., Messner, S.J., Meschino, W.S., Pirod, C.A., Narod, S.A.: Surveillance of BRCA1 and BRCA2 mutation carriers with magnetic resonance imaging, ultrasound, mammography, and clinical breast examination. Journal of the American Medical Association 292(11) (2004) 1317-1325 
2. American College of Radiology(ACR): Breast Imaging Reporting and Data System (BI-RADS) (2nd edn.). American College of Radiology, Reston, Virginia (1995)

3. Kuhl, C.K., Mielcareck, P., Klaschik, S., Leutner, C., Wardelmann, E., Giesek, J., Schil, H.H.: Dynamic breast mr imaging: Are signal intensity time course data useful for differential diagnosis of enhancing lesions? Radiology 211 (1999) 101-110

4. Zuo, C., Jiang, A., Buff, B.L., Mahon, T.G., Wong, T.Z.: Automatic motion correction for breast $\mathrm{mr}$ imaging. Radiology 198(3) (1996) 903-906

5. Kumar, R., Asmuth, J.C., Hanna, K., Bergen, J., Hulka, C., Kopans, D.B., Weisskoff, R., Moore, R.: Application of 3d registration for detecting lesions in magnetic resonance breast scans. Proc. SPIE Medical Imaging 1996: Image Processing 2710 (1996) 646-656

6. Davis, M., Khotanzad, A., Flamig, D., Harms, S.: A physics based coordinate transformation for 3d image matching. IEEE Trans. Med. Imag. 16(3) (1997) $317-328$

7. Rueckert, D., Sonoda, L.I., Hayes, C., Hill, D.L.G., Leach, M.O., Hawkes, D.J.: Nonrigid registration using free-form deformations: application to breast MR images. IEEE Trans. Med. Imag. 18(8) (1999) 712-721

8. Schnabel, J.A., Tanner, C., Castellano-Smith, A.D., Degenhard, A., Leach, M.O., Hose, D.R., Hill, D.L.G., Hawkes, D.J.: Validation of nonrigid image registration using finite-element methods: Application to breast mr images. IEEE Trans. Med. Imag. 22(2) (2003) 238-247

9. Rohlfing, T., Jr., C.R.M., Bluemke, D.A., Jacobs, M.A.: Volume-preserving nonrigid registration of $\mathrm{mr}$ breast images using free-form deformation with an incompressibility constraint. IEEE Trans. Med. Imag. 22(6) (2003) 730-741

10. Crum, W.R., Tanner, C., Hawkes, D.J.: Anisotropic multi-scale fluid registration: evaluation in magnetic resonance breast imaging. Physics in Medicine and Biology 50(21) (2005) 5153-5174

11. Gennert, M.A., Negahdaripour, S.: Relaxing the brightness constancy assumption in computing optical flow. Massachusetts Institute of Technology Artificial Intelligence Laboratory AIM-975 (1987)

12. Horn, B.K., Schunck, B.G.: Determining optical flow. Artificial Intelligence $\mathbf{1 7}$ (1981) 185-204

13. Barber, D.C.: Registration of low resolution medical images. Physics in Medicine and Biology 37(7) (1992) 1485-1498

14. Hartkens, T., Rueckert, D., Schnabel, J., Hawkes, D., Hill, D.: VTK CISG registration toolkit: An open source software package for affine and non-rigid registration of single- and multimodal 3d images. In: Proc. Bildverarbeitung in der Medizin 2002. LNCS, Leipzig, Springer Verlag (2002) 409-412 